\title{
A UTILIZAÇÃO DO QUESTIONÁRIO HHIE-S ASSOCIADO À AVALIAÇÃO AUDIOLÓGICA E VOCAL NUM GRUPO DE IDOSOS
}

\author{
Mauriceia Cassol \\ Maria Inês Dornelles da Costa Ferreira** \\ Deise Maria de Azevedo Poglia***
}

\section{Resumo}

Este estudo objetiva identificar a importância do questionário Hearing Handicap Inventory for the Elderly - Screening Version (HHIE-S) como indicador da necessidade de avaliação audiológica em um grupo de idosos, relacionando as perdas auditivas às possíveis alterações vocais. Fizeram parte deste estudo cinco indivíduos idosos do gênero feminino, na faixa etária de 57 a 75 anos. Primeiramente, foi aplicado o questionário HHIE-S seguido da avaliação audiológica básica e da avaliação perceptivo-auditiva da voz. Nos resultados, os cinco indivíduos idosos apresentaram alteração em um ou mais aspectos vocais. As alterações das características vocais puderam ser correlacionadas às perdas auditivas em três dos cinco casos. Salienta-se a importância da avaliação vocal e da utilização de escalas de auto-avaliação, como o questionário HHIE-S, como indicadores da identificação precoce da deficiência auditiva.

Palavras-chave: Perda Auditiva. Presbiacusia. Saúde do Idoso. Qualidade Vocal.

\section{Introdução}

A expectativa de vida tem aumentado consideravelmente em todo o mundo, graças aos avanços das ciências da saúde, que permitem a manutenção de diversas funções orgânicas e a restauração de grande parte das funções que já não ocorrem de forma adequada (MITRE, 2001). A população idosa brasileira tem-se ampliado rapidamente, sendo que a fai-

* Coordenadora e Professora do curso de Fonoaudiologia da Fundação Universidade Federal de Ciências da Saúde de Porto Alegre (UFSCPA); Doutora em Ciências da Saúde em Geriatria (PUCRS); Especialista em Voz pelo Centro de Estudos da Voz (CEV). E-mail: mcassol@terra.com.br

** Professora do curso de Fonoaudiologia do Centro Universitário Metodista (IPA); Doutora em Letras, Lingüística Aplicada (PUCRS); Especialista em Audiologia pelo CFFa. E-mail: costa.ferreira@terra.com.br

*** Fonoaudióloga pelo Centro Universitário Metodista (IPA). E-mail: poglinha@yahoo.com.br 
xa etária a partir de 60 anos é a que mais cresce. No período entre 1950 e 2025, segundo as projeções estatísticas da Organização Mundial de Saúde (OMS), o grupo de idosos no Brasil deverá ter aumentado em 15 vezes, enquanto a população total em cinco. O País ocupará, assim, o sexto lugar quanto ao contingente de idosos, alcançando, em 2025, cerca de $32 \mathrm{mi}-$ lhões de pessoas com 60 anos ou mais de idade. O idoso é responsável pelo maior consumo na área da saúde, sendo que as internações hospitalares são mais freqüentes, e o tempo de ocupação do leito é maior do que o de outras faixas etárias. As doenças crônicas e múltiplas perduram por vários anos e exigem acompanhamento médico e de equipes multidisciplinares permanentes e intervenções contínuas (BRASIL, 1999).

Dentro dessa perspectiva, busca-se desenvolver trabalhos com o objetivo de proporcionar melhor qualidade de vida ao idoso. Esta pesquisa teve como objetivo identificar a importância do questionário HHIE-S como indicador da necessidade de avaliação audiológica em idosos, relacionando as perdas auditivas às possíveis alterações vocais.

\subsection{Presbiacusia}

A perda auditiva relativa à idade é definida como o prejuízo auditivo associado a vários tipos de disfunções auditivas, periférica e central, que acompanham a idade sem levar em conta traumas acústicos, genéticos e condições patológicas. O termo prejuízo auditivo implica em deficit absoluto da percepção limiar e supraliminar (BARALDI, 2005).

As modificações ocorrem na maioria das estruturas e órgãos, sendo absolutamente fisiológicas (BESS; WILLIAMS; LICHTENSTEIN, 2001). Por isso, devemos destacar a deficiência auditiva e o envelhecimento da voz como aspectos importantes a serem considerados nesta etapa da vida, pois constituem-se em distúrbios de comunicação que impedem o idoso de desempenhar seu papel na sociedade por apresentar mudanças na percepção, motivação, memória e características psicossociais. Junto a esses fatores também encontramos a diminuição na compreensão da fala, que pode levar o idoso ao isolamento da sociedade.

O fonoaudiólogo é o profissional cuja função, entre outras, é o desenvolvimento da comunicação em vários aspectos contribuindo para a re-inserção do idoso na sociedade (RUSSO; ALMEIDA; FREIRE, 2003). Em caso de perda auditiva e alterações vocais, o fonoaudiólogo será res- 
ponsável pela seleção, indicação e adaptação de próteses auditivas com o objetivo de recuperar as necessidades acústicas reveladas pela configuração audiométrica. Além disso, orientará o idoso no que se refere ao processo de reabilitação auditiva e vocal (BESS; WILLIAMS; LICHTENSTEIN, 2001).

A presbiacusia é uma das conseqüências mais importantes do envelhecimento. É o decréscimo fisiológico da audição com a idade, no qual todas as estruturas do organismo se modificam, influenciando diretamente a qualidade da audição e compreensão, pois há o envelhecimento das orelhas externa, média e interna, e das vias auditivas centrais. Apesar disso, a deficiência auditiva decorrente de presbiacusia está apenas centralizada nas alterações de orelha interna.

Tal distúrbio caracteriza-se pelo rebaixamento dos limiares audiométricos para as freqüências altas, acima de $1.000 \mathrm{~Hz}$, o que dificulta a percepção dos sons consonantais, principalmente em ambientes ruidosos reduzindo, assim, a inteligibilidade da fala.

A prevalência da presbiacusia é de $12 \%$ entre os 45 e 64 anos, sobe para $24 \%$ entre as idades de 65 e 74 anos, e aumenta para $39 \%$ nas idades acima de 75 anos (CALAIS et al. 2005).

A maioria das consoantes do português brasileiro situa-se entre $1000 \mathrm{~Hz}$ a $6000 \mathrm{~Hz}$ e $15 \mathrm{dBNA}$ a $30 \mathrm{dBNA}$. Em caso de presbiacusia, o idoso perde as diferenças acústicas mínimas da fala, o que dificultará a compreensão da mesma (BEHLAU et al. 2001).

A inteligibilidade da fala está relacionada, principalmente, à identificação do ponto articulatório dos sons, que pode ser influenciada pela resolução temporal que é a capacidade que o sistema auditivo tem de detectar a ocorrência de dois eventos auditivos consecutivos. Se os indivíduos idosos apresentarem piora na resolução temporal, tal fato poderá acarretar na dificuldade em reconhecer e discriminar o ponto articulatório dos sons, prejudicando a inteligibilidade da fala (BESS; WILLIAMS; LICHTENSTEIN, 2001).

A discriminação do ponto articulatório diminui com o aumento da idade e está relacionada ao processamento auditivo dos idosos. Os aspectos temporais da audição atuam diretamente na capacidade do ouvinte em reconhecer, discriminar e perceber as modificações acústicas da fala, sendo influenciado pela maturação da via auditiva, pelos desenvolvimentos lingüístico, psíquico e cognitivo dos indivíduos. A interação adequada 
destes sistemas permitirá ao ouvinte perceber e compreender a mensagem transmitida pelo falante (BALEN; SANTOS, 1998).

Nesse caso, a resolução temporal pode ser influenciada pela idade e pela perda auditiva periférica.

A presbiacusia sensorial refere-se à atrofia do Órgão de Corti, o que justificaria a perda auditiva para freqüências altas, que torna a percepção dos sons consonantais muito difícil, principalmente quando a comunicação ocorre em ambientes ruidosos (RUSSO, 2004). Já a presbiacusia neural é definida pela perda de fibras nervosas no sistema nervoso central, que, além da deficiência auditiva para freqüências altas, gera maior comprometimento na discriminação. O tipo metabólico reflete alterações nos processos biofísicos e bioquímicos envolvidos nos mecanismos de transdução da orelha interna, refletindo dificuldades no reconhecimento de palavras. Por fim, o tipo mecânico sugere a alteração da função da membrana basilar, gerando perda auditiva progressiva nas freqüências altas e conseqüente dificuldade no reconhecimento da fala (SANTOS; RUSSO, 1993).

A queixa principal dos indivíduos idosos portadores de presbiacusia é a dificuldade em compreender a fala, geralmente acompanhada de ruído. Esse quadro pode ser acompanhado de zumbido, alteração da função auditiva central e perda de memória (RUSSO, 2004).

\subsection{Avaliação Audiológica}

$\mathrm{Na}$ intenção de avaliar o sucesso do processo de seleção, indicação e adaptação de próteses auditivas, alguns questionários foram propostos. Dentre eles, destaca-se o Hearing Handicap Inventory for the Elderly (HHIE) ${ }^{1}$. O referido questionário é, também, pesquisado pela comunidade científica fonoaudiológica, na sua relação com os limiares auditivos tonais. Acredita-se que esse possa ser um indicador da necessidade de uma avaliação audiológica e vocal.

\footnotetext{
${ }^{1}$ Elaborado por Ventry e Weinstein em 1982 e adaptado para o português por WIESELBERG, 1997. Ver também: MATAS, Carla Gentile; Iório, Maria Cecília Martinelli. Verificação e Validação do Processo de Seleção e Adaptação de Próteses Auditivas. In: ALMEIDA, Kátia de; IÓRIO, Maria Cecília Martinelli. Próteses Auditivas: fundamentos teóricos e aplicações clínicas. São Paulo: Lovise, 2003. P. 328.
} 


\subsection{Presbifonia}

A máxima eficiência vocal ocorre dentre os 25 e 40 anos, dependendo da saúde física e psicológica, história de vida, raça, hereditariedade e fatores ambientais e sociais (SUZUKI, 2003).

Os dois principais componentes alterados devido ao processo de envelhecimento da laringe são as cartilagens e os músculos, através da atrofia muscular pela redução de massa, edema, desidratação da mucosa e pela calcificação das cartilagens, que levam à progressiva redução da sua mobilidade. Observa-se um aumento da freqüência fundamental nos homens e, nas mulheres, um abaixamento da freqüência fundamental, tornando cada vez mais as vozes parecidas (MITRE, 2001). Outro fator importante a ser analisado é a presbifonia, na qual ocorre uma flacidez da musculatura orofacial da laringe, das pregas vocais, enrijecimento das articulações, redução dos tempos de expiração e fonação, prejudicando a qualidade e o desempenho vocal, profissional e social, e que deve ser compreendida como parte do processo de envelhecimento e não como um distúrbio vocal (SUZUKI, 2003).

\subsection{Avaliação da Voz}

A avaliação perceptivo-auditiva é a melhor forma de avaliar a qualidade vocal e tem como principal objetivo definir as principais características vocais, ou seja, rouca, áspera, soprosa, astênica, tensa, pastosa, trêmula, sussurrada, fluída, bitonal, diplofônica, monótona, infantilizada, presbifônica, hipernasal e hiponasal. O julgamento da qualidade da voz é primeiramente perceptivo, embora suas propriedades possam, ainda, ser examinadas em nível fisiológico e acústico (BARROS; ANGELIS, 2002). A avaliação do comportamento vocal consiste em uma análise dos principais parâmetros utilizados pelo paciente na comunicação habitual. Os parâmetros mais importantes dessa avaliação são: qualidade vocal (tipos de vozes), intensidade vocal, ressonância, tempos máximos de fonação, freqüência, velocidade da fala, articulação, ritmo, resistência vocal, dinâmica respiratória, estrutura fonoarticulatória, avaliação corporal e psicodinâmica vocal (BEHLAU; PONTES, 1995). 


\section{Metodologia}

Esta pesquisa foi desenvolvida no Centro Universitário Metodista IPA. O grupo avaliado constou de cinco indivíduos idosos na faixa etária de 57 a 75 anos, do gênero feminino. Todas freqüentam as oficinas de Fonoaudiologia da Universidade do Adulto Maior (UAM), em encontros semanais de uma hora de duração. Os critérios de exclusão da amostra adotados foram: participante com infecção respiratória aguda no dia da avaliação, doença neurológica prévia (AVC, Parkinson, Demência), indivíduos com cirurgia de cabeça e pescoço prévia e todos aqueles que não concordaram em participar da pesquisa. Convém ressaltar a participação de 1 indivíduo com 57 anos de idade que não pertence à denominação idoso, proposta pelo Estatuto do Idoso (BRASIL, 2003), Artigo $1^{\circ}$. O referido sujeito não foi excluído do estudo por apresentar o mesmo perfil que os demais sujeitos.

O projeto do estudo foi aprovado pelo Comitê de Ética em Pesquisa do Centro Universitário Metodista IPA, com protocolo de registro $\mathrm{n}^{\circ}$ 1076.

A pesquisadora comprometeu-se em manter confidencialidade e sigilo em relação aos dados coletados, vinculados aos nomes dos participantes da pesquisa. Após o esclarecimento quanto aos objetivos e metodologia do trabalho, os idosos participantes assinaram um termo de consentimento informado, o qual ressalta a inexistência de desconfortos ou riscos aos mesmos e descreve os procedimentos empregados na pesquisa.

A avaliação perceptivo-auditiva da voz foi realizada no primeiro dia de participação dos idosos na oficina. O material de fala colhido para a avaliação foi o emprego da emissão da vogal [a] (Anexo A), solicitando-se que o sujeito inspirasse e emitisse o referido som, em uma única freqüência, sem variação musical ou de intensidade, ou seja, que fosse emitido de modo habitual. Logo em seguida, foi solicitada a realização da contagem de números de 1 a 20. Para a coleta dos dados, os sujeitos foram instruídos a permanecer em pé, com os braços estendidos ao longo do corpo, e o microfone foi posicionado em um ângulo de $90^{\circ}$ da boca do indivíduo. Para emissão da vogal foi mantida uma distância em torno de $5 \mathrm{~cm}$, para evitar interferências no sinal e manter uma proporção sinal-ruído elevado. Para a fala encadeada foi mantida uma distância maior, de $10 \mathrm{~cm}$, para que o ruído respiratório não contaminasse a gravação (BEHLAU et al., 2001) Na 
coleta do material de voz, foram utilizados um gravador digital (Portable MiniDisc Recorder MZ-R3) da marca SONY e um microfone profissional, condensador, estéreo, omnidirecional, sensibilidade $-35 \mathrm{~dB}$, da marca $L E$ SON modelo MP66. As gravações foram realizadas em ambiente silencioso, porém sem isolamento acústico. Não foram medidos os níveis de ruído da sala. As vozes foram avaliadas e analisadas com o auxílio de duas fonoaudiólogas professoras do curso de fonoaudiologia do Centro Universitário Metodista IPA, e uma aluna bolsista de iniciação científica. As examinadoras receberam um protocolo, contendo os itens a serem avaliados. As vozes foram apresentadas, aleatoriamente, e o julgamento de cada voz foi realizado individualmente.Todos os participantes da pesquisa foram encaminhados para uma consulta com um médico otorrinolaringologista.

A avaliação audiológica foi realizada no laboratório de Audiologia das Clínicas Integradas do Centro Universitário Metodista - IPA, localizada no Hospital Parque Belém. Os equipamentos utilizados foram: imitanciômetro AZ7 e Audiômetro AC33, ambos da marca Interacoustics devidamente calibrados de acordo com as normas vigentes.

A avaliação audiológica básica iniciou-se com a imitanciometria, objetivando a exclusão dos indivíduos que apresentassem alterações de orelha externa e/ou média. Em seguida, realizou-se a audiometria tonal e vocal em que a classificação utilizada para a determinação do padrão de normalidade e para o grau de perda auditiva foi a proposta por Davis e Silverman (1970). A audiometria vocal foi realizada a viva-voz. Posteriormente, realizou-se a aplicação do questionário HHIE-S (Anexo B) para analisar a percepção do handicap auditivo do idoso. Tal questionário foi aplicado individualmente, pelo mesmo pesquisador, e as perguntas foram lidas e explicadas para os sujeitos do estudo. A análise do questionário foi realizada de acordo com o valor dos itens do questionário que foram classificados em sim (10 pontos), não (nenhum ponto) e às vezes (5 pontos) (MATAS; IÓRIO, 2003). Dessa forma, pontuações inferiores a $16 \%$ indicam não haver percepção do handicap; de $18 \%$ a 42\%, o indivíduo é classificado como tendo uma percepção de leve a moderada; e acima de $42 \%$, uma percepção moderada.

Os dados foram analisados qualitativamente correlacionando os resultados obtidos na avaliação perceptivo-auditiva da voz com a avaliação audiológica e aplicação do questionário HHIE-S. 


\section{Resultados}

Os resultados obtidos na avaliação auditiva constam na tabela 1 .

Tabela 1 - Resultados da Aplicação do Questionário HHIE Associado à Avaliação Audiológica

\begin{tabular}{|c|c|c|c|c|c|c|c|c|}
\hline \multirow{2}{*}{$\stackrel{\frac{2}{0}}{\underline{\underline{c}}}$} & \multirow{2}{*}{$\begin{array}{l}\frac{0}{0} \\
\frac{\pi}{0} \\
-\end{array}$} & \multicolumn{2}{|c|}{$\begin{array}{c}\text { Audiometria } \\
\text { Tonal }\end{array}$} & \multicolumn{2}{|c|}{ LRF } & \multicolumn{2}{|c|}{$\begin{array}{c}\text { IRF } \\
\text { monossílabos }\end{array}$} & \multirow{2}{*}{$\begin{array}{c}\text { HHI E-S e } \\
\text { grau de } \\
\text { percepção } \\
\text { do handicap }\end{array}$} \\
\hline & & OD & $\mathrm{OE}$ & OD & $\mathrm{OE}$ & OD & $\mathrm{OE}$ & \\
\hline 1 & 57 & Normal & Normal & $25 \mathrm{~dB}$ & $20 \mathrm{~dB}$ & $100 \%$ & $100 \%$ & LAN - 0\% \\
\hline 2 & 75 & Neuros. & Neuros. & $45 \mathrm{~dB}$ & $30 \mathrm{~dB}$ & $92 \%$ & $92 \%$ & $\begin{array}{l}\text { Percepção } \\
\text { Moderada - } \\
40 \%\end{array}$ \\
\hline 3 & 64 & Normal & Normal & $20 \mathrm{~dB}$ & $\mathrm{OdB}$ & $100 \%$ & $100 \%$ & LAN - $10 \%$ \\
\hline 4 & 75 & \begin{tabular}{|c|} 
Restrita \\
freq. \\
Altas \\
\end{tabular} & \begin{tabular}{|l} 
Restrita \\
freq. \\
Altas \\
\end{tabular} & $25 \mathrm{~dB}$ & $25 \mathrm{~dB}$ & $92 \%$ & $100 \%$ & $\begin{array}{c}\text { Não Percebe } \\
-10 \%\end{array}$ \\
\hline 5 & 75 & \begin{tabular}{|c|} 
Restrita \\
freq. \\
Altas
\end{tabular} & Neuros. & $25 \mathrm{~dB}$ & $25 \mathrm{~dB}$ & $92 \%$ & $56 \%$ & $\begin{array}{l}\text { Percepção } \\
\text { Moderada - } \\
40 \%\end{array}$ \\
\hline
\end{tabular}

Fonte: Elaborada pelos Autores com base nos dados obtidos na pesquisa.

$\mathrm{Na}$ análise dos testes de audiometria tonal, dois dos indivíduos $\left(\mathrm{n}^{\mathrm{o}}\right.$ 1 e $n^{\circ} 3$ ) apresentaram limiares auditivos normais para ambas as orelhas, índice de reconhecimento de fala de $100 \%$, limiar de reconhecimento de fala compatível com a média das freqüências de $500 \mathrm{~Hz}, 1000 \mathrm{~Hz}$ e $2000 \mathrm{~Hz}$. Ambos não referiram queixas no questionário HHIE-S ( 0 pontos ou $0 \%$ de percepção do handicap). Um indivíduo $\left(\mathrm{n}^{\circ} 2\right)$ apresentou perda auditiva neurossensorial para ambas as orelhas, limiares de reconhecimento de fala (LRF) de $45 \mathrm{~dB}$ para orelha direita e $30 \mathrm{~dB}$ para a orelha esquerda e índice de reconhecimento de fala (IRF) de $92 \%$ para ambas as orelhas. No questionário HHIE-S, obteve percepção moderada de seu handicap auditivo $(40 \%)$. O indivíduo $\left(n^{\circ} 4\right)$ apresentou perda auditiva restrita às freqüências altas para ambas as orelhas, LRF de $25 \mathrm{~dB}$ para ambas as orelhas e IRF de $92 \%$ para a orelha direita e $100 \%$ para a orelha esquerda. No questionário HHIE, referiu queixas com relação ao handicap auditivo (10\%). O indivíduo $\left(\mathrm{n}^{\mathrm{o}} 5\right)$ apresentou perda auditiva restrita às freqüências altas para a orelha direita e perda auditiva neurossensorial para a orelha esquerda com 
LRF de $25 \mathrm{~dB}$ para ambas as orelhas e IRF de $92 \%$ para a orelha direita e $56 \%$ para a orelha esquerda. Apresentou percepção moderada de seu handicap auditivo (40\%) no questionário HHIE.

Partindo de uma amostra de cinco vozes de indivíduos idosos, foi realizada a análise perceptivo-auditiva, e os resultados encontrados para os parâmetros de articulação, pitch, intensidade, ressonância, qualidade vocal, velocidade da fala e ataque vocal estão descritos no quadro 1.

Quadro 1 - Resultados da Avaliação Vocal Através da Análise Perceptivo-Auditiva

\begin{tabular}{|c|c|c|c|c|c|c|c|}
\hline 릉 & Articul. & Pitch & I ntens. & $\begin{array}{l}\text { Resso- } \\
\text { nância }\end{array}$ & $\begin{array}{c}\text { Qualid. } \\
\text { vocal }\end{array}$ & $\begin{array}{l}\text { Veloc. } \\
\text { de fala }\end{array}$ & $\begin{array}{c}\text { Ataque } \\
\text { vocal }\end{array}$ \\
\hline 1 & Normal & Normal & Adequada & $\begin{array}{l}\text { Laringo } \\
\text { Faríngea }\end{array}$ & Adaptada & Normal & Brusco \\
\hline 2 & Normal & Grave & Adequada & $\begin{array}{l}\text { Laringo } \\
\text { Faríngea }\end{array}$ & $\begin{array}{l}\text { Rouca } \\
\text { Leve }\end{array}$ & Reduz. & Normal \\
\hline 3 & Normal & Normal & Adequada & Equil. & $\begin{array}{l}\text { Rouca } \\
\text { Leve }\end{array}$ & Normal & Brusco \\
\hline 4 & Normal & Grave & Aument. & $\begin{array}{l}\text { Laringo } \\
\text { Faríngea }\end{array}$ & $\begin{array}{l}\text { Rouca } \\
\text { Leve }\end{array}$ & Normal & Brusco \\
\hline 5 & Normal & Grave & Reduzida & $\begin{array}{l}\text { Laringo } \\
\text { Faríngea }\end{array}$ & $\begin{array}{c}\text { Rouca } \\
\text { moderada } \\
\text { Soprosa e } \\
\text { áspera } \\
\text { leve } \\
\text { trêmula }\end{array}$ & Normal & Brusco \\
\hline
\end{tabular}

Fonte: Elaborado pelos Autores com base nos dados obtidos na pesquisa.

Todos os idosos avaliados apresentaram tipo articulatório normal;

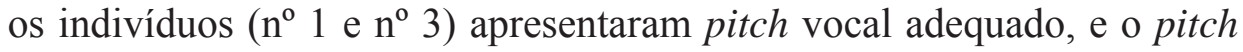
grave foi encontrado nos sujeitos $\left(\mathrm{n}^{\mathrm{o}} 2,4\right.$ e 5$)$. A intensidade vocal adequada foi encontrada em três indivíduos $\left(\mathrm{n}^{\circ} 1,2\right.$ e 3$)$, sendo que o indivíduo $\left(\mathrm{n}^{\mathrm{o}}\right.$ 4) apresentou intensidade vocal aumentada, e o indivíduo $\left(n^{\circ} 5\right)$, intensidade vocal reduzida. Na avaliação da ressonância vocal, os indivíduos $\left(\mathrm{n}^{\circ} 1\right.$, 2,4 e 5) apresentaram foco de ressonância laringo-faríngica, e o sujeito $\left(\mathrm{n}^{\mathrm{o}}\right.$ 3 ) foco de ressonância equilibrado. Na análise da qualidade vocal, os indivíduos $\left(\mathrm{n}^{\mathrm{o}} 2,3\right.$ e 4$)$ apresentaram rouquidão em grau leve. O sujeito $\left(\mathrm{n}^{\mathrm{o}} 1\right)$ apresentou voz do tipo adaptada, e o sujeito $\left(\mathrm{n}^{\circ} \mathrm{5}\right)$, qualidade vocal do tipo rouca de grau moderado, soprosa e aspereza de grau leve, com presença de tremor também em grau leve. 


\section{Discussão}

Como vimos na literatura, a perda auditiva relativa à idade pode favorecer o indivíduo para que apresente um deficit significativo na comunicação. Paralelo a este acontecimento, ocorre o impacto no envelhecimento vocal (BEHLAU et al., 2001).

A partir dos resultados relatados nesta pesquisa, demonstrados na tabela 1 e quadro 1 , os indivíduos ( $n^{\circ} 1$ e 3 ) não apresentaram percepção do handicap auditivo para o questionário HHIE e obtiveram limiares auditivos normais na audiometria tonal em ambas as orelhas. Também apresentaram resultados normais na avaliação vocal. Os indivíduos ( $\mathrm{n}^{\circ} 4$ e 5$)$ apresentaram presbiacusia, caracterizada pelo rebaixamento nos limiares auditivos para as freqüências altas. Esta alteração é caracterizada pela dificuldade na percepção dos sons consonantais, principalmente em ambiente ruidoso, reduzindo, assim, a inteligibilidade da fala (CALAIS et al., 2005; SANTOS; RUSSO, 1993). O indivíduo ( $\left.n^{\circ} 4\right)$ não apresentou percepção no handicap auditivo no questionário e o indivíduo $\left(\mathrm{n}^{\circ} 5\right)$ apresentou percepção moderada (RUSSO; ALMEIDA; FREIRE, 2003). Além disso, esses indivíduos mostram alterações na qualidade vocal caracterizada por modificação no pitch, intensidade, ressonância, qualidade vocal, velocidade de fala e ataque vocal, decorrente do processo de envelhecimento das estruturas vocais (SUZUKI, 2003).

A voz presbifônica é uma voz com grau variado de deterioração, que se expressa na falta de sustentação de freqüência, intensidade e qualidade de emissão, sendo constantes as quebras de sonoridade. Tal característica é observada nos indivíduos idosos, com alterações mais evidentes após os 65 anos de idade (BEHLAU et al., 2001) O indivíduo ( $\left.\mathrm{n}^{\mathrm{o}} 2\right)$ apresentou percepção moderada para o questionário HHIE. Em relação à avaliação vocal, houve alteração nos parâmetros de pitch, ressonância, qualidade vocal, velocidade de fala e ataque vocal (BESS; WILLIAMS; LICHTENSTEIN, 2001).

\section{Conclusão}

Por meio deste estudo, observou-se que os indivíduos com limiares auditivos normais (LAN) não apresentaram queixas no questionário HHIE-S, apresentando resultados adequados a todos os parâmetros tes- 
tados na avaliação vocal. Os sujeitos que apresentaram resultados alterados na avaliação audiológica básica e percepção do handicap auditivo no questionário HHIE apresentaram mais alterações nos padrões vocais, pois o monitoramento vocal é basicamente realizado pela via auditiva, que deve encontrar-se preservada para que os parâmetros vocais não sejam alterados. Convém ressaltar que os resultados obtidos neste estudo não permitem generalizações em função do tamanho reduzido da amostra e da inexistência de análise estatística. Com esta pesquisa, podemos concluir que a aplicação do questionário HHIE-S é útil na prática clínica fonoaudiológica na atuação com indivíduos idosos.

\section{USE OF THE HHIE-S QUESTIONNAIRE ASSOCIATED WITH VOCAL AND AUDIOLOGICAL EVALUATION IN THE ELDERLY GROUP}

\section{Abstract}

This study aimed at identifying the importance of the Hearing Handicap Inventory for the Elderly - Screening Version (HHIE-S) questionnaire as an indicator of the need for an audiological evaluation in a group of elderly, and verifying the relation between hearing loss and possible vocal change in 5 elderly females with ages ranging from 57 to 75 years. The HHIE-S questionnaire was applied, followed by a basic audiological evaluation and auditory-perceptive vocal evaluation. In the results, 5 elderly individuals presented changes in one or more vocal aspects. The changes in vocal characteristics may be correlated to hearing loss in 3 of the 5 cases. The importance of vocal evaluation and use of self-evaluation scales as the HHIE-S questionnaire as indicators for early identification of hearing impairment is highlighted.

Keywords: Hearing Loss. Presbycusis. Aging. Health. Voice Quality.

\section{REFERÊNCIAS}

BALEN, Sheila Andreoli; SANTOS, Teresa Maria Momenshon dos. Aspectos Temporais da Audição e Percepção Acústica da Fala: revisão da literatura. In: BEVILACQUA, Maria Cecilia (Org.). Audiologia Atual. Sao Paulo: Frontis Editorial, 1998. P. 57-82. 
BARALDI, Giovana dos Santos; BORGES, Alda Christina Lopes de Caravalho; ALMEIDA, Lais Castro de. Avaliação Supraliminar da Fala em Indivíduos Idosos. In: ENCONTRO INTERNACIONAL DE AUDIOLOGIA, 20., São Paulo. Anais... São Paulo: Academia Brasileira de Audiologia, 2005. 1 CD-ROM.

BARROS, Ana Paula Brandão; ANGELIS, Elisabete Carrara de. Análise Acústica da Voz. In: DEDIVITIS, Rogério Aparecido; BARROS, Ana Paula Brandão (Org.). Métodos de Avaliação e Diagnóstico de Laringe e Voz. São Paulo: Lovise, 2002. P. 201-221.

BEHLAU, Mara Suzana et al. Avaliação de Voz. In: BEHLAU, Mara Suzana (Org.). Voz: o livro do especialista. Rio de Janeiro: Revinter, 2001. P. 85-172.

BEHLAU, Mara Suzana; PONTES, Paulo Augusto de Lima. Avaliação e Tratamento das Disfonias. São Paulo: Lovise, 1995.

BESS, Fred; WILLIAMS, Andrea Hedley, LICHTENSTEIN Michael. A Avaliação Audiológica dos Idosos. In: MUSIEK Frank E.; RINTELMANN William F. Perspectivas Atuais em Avaliação Audiológica. São Paulo: Manole, 2001. P. 343-354.

BRASIL. Lei n. 10.741, de 1 de outubro de 2003. Estatuto do Idoso. Diário Oficial [da] República Federativa do Brasil, Brasília, DF, 3 out. 2003. Disponível em: $<$ http://www.planalto.gov.br/ccivil/LEIS/2003/L10.741.htm\#art118>. Acesso em: 20 jan. 2008.

BRASIL. Ministério da Saúde. Portaria 1395/GM. Dispõe sobre a aprovação da Política Nacional de Saúde do Idoso. Diário Oficial [da] República Federativa do Brasil, Brasília, DF, 1999. Disponível em: <http://www.ufrgs.br/3idade/portaria1395gm.html $>$. Acesso em : 10 jun. 2005.

CALAIS, Lucila Leal et al. Acuidade e Sensibilidade Auditiva no Idoso. In: ENCONTRO INTERNACIONAL DE AUDIOLOGIA, 20., São Paulo. Anais... São Paulo: Academia Brasileira de Audiologia, 2005. 1 CD-ROM.

DAVIS, Hallowell; SILVERMAN, S. Richard. Hearing and Deafness. 3rd ed. New York: Holt, Rinehart and Winston, 1970. 
MATAS, Carla Gentile; IÓRIO, Maria Cecília Martinelli. Verificação e Validação do Processo de Seleção e Adaptação de Próteses Auditivas. In: ALMEIDA, Katia de; IORIO, Maria Cecília Martinelli. Próteses Auditivas: fundamentos teóricos e aplicações clínicas. São Paulo: Lovise, 2003. P. 305-334.

MITRE, Edson Ibrahim. Otorrinolaringologia e Fonoaudiologia. São José dos Campos: Pulso, 2001.

RUSSO, Ieda Chaves Pacheco. Distúrbios da Audição. In: . Intervenção Fonoaudiológica na Terceira Idade. Rio de Janeiro: Revinter; 2004. P. 51-82.

RUSSO, Ieda Chaves Pacheco; ALMEIDA, Katia de; FREIRE, Katya Guglielmi Marcondes. Seleção e Adaptação da Prótese Auditiva para o Idoso. In: ALMEIDA, Katia de; IORIO, Maria Cecília Martinelli. Próteses Auditivas: fundamentos teóricos e aplicações clínicas. São Paulo: Lovise, 2003. P. 24-27.

SANTOS, Teresa Momensohn dos; RUSSO, Ieda Chaves Pacheco. A Prática da Audiologia Clínica. São Paulo: Cortez, 1993.

SUZUKI, Heloisa Sawada. Conhecimentos Essenciais para Atender Bem o Paciente Idoso. São José dos Campos: Pulso, 2003.

WIESELBERG, Margarita Bernal. AAuto Avaliação do Handicap em Idosos Portadores de Deficiência Auditiva: o uso do HHIE. 1997. 109 f. Dissertação (Mestrado em Fonoaudiologia)-Departamento de Fonoaudiologia, Pontifícia Universidade Católica de São Paulo, São Paulo, 1997. 


\section{APÊNDICE A \\ Protocolo de Registro da Avaliação Perceptivo-auditiva da Voz}

1.Tipo de voz / Grau de alteração:

2.Ataque vocal:

isocrônico ( ) brusco ( ) alternado ( )

3.Articulação:

normal ( ) hipotônica ( ) hipertônica ( ) exagerada ( )

4.Velocidade de fala:

normal ( ) reduzida ( ) aumentada ( ) variada ( )

5.Intensidade:

adequada ( ) aumentada ( ) reduzida ( )

6. Pitch:

normal ( ) agudo ( ) grave ( )

7. Ressonância:

equilibrada ( ) laríngea ( ) faríngea ( ) laringo-faríngea ( ) nasal ( ) 


\section{ANEXO A - HHIE-S}

\section{HHIE-S \\ The Hearing Handicap Inventory for the EIderly \\ Screening Version}

Nome:

Data do exame:

Sexo: ( )M ( )F Idade:....

$\mathrm{DN}$ :

\begin{tabular}{|l|l|l|l|l|}
\hline \multicolumn{2}{|l|}{} & Sim & $\begin{array}{c}\text { Às } \\
\text { Vezes }\end{array}$ & Não \\
\hline E-1 & $\begin{array}{l}\text { A dificuldade em ouvir faz você se sentir constrangido } \\
\text { ou sem jeito quando é apresentado a pessoas desconhe- } \\
\text { cidas? }\end{array}$ & & & \\
\hline E-2 & $\begin{array}{l}\text { A dificuldade em ouvir faz você se sentir frustrado ou } \\
\text { insatisfeito quando conversa com pessoas de sua famí- } \\
\text { lia? }\end{array}$ & & & \\
\hline S-3 & $\begin{array}{l}\text { Você sente dificuldade em ouvir quando alguém fala co- } \\
\text { chichando? }\end{array}$ & & & \\
\hline E-4 & $\begin{array}{l}\text { Você sente prejudicado em função de seu problema au- } \\
\text { ditivo? }\end{array}$ & & & \\
\hline S-5 & $\begin{array}{l}\text { A diminuição da audição lhe causa dificuldades quando } \\
\text { visita amigos, parentes ou vizinhos? }\end{array}$ & & & \\
\hline S-6 & $\begin{array}{l}\text { A dificuldade em ouvir faz com que você vá a serviços } \\
\text { religiosos menos vezes do que gostaria? }\end{array}$ & & & \\
\hline E-7 & $\begin{array}{l}\text { A dificuldade em ouvir faz você ter discussões ou brigas } \\
\text { com sua família? }\end{array}$ & & & \\
\hline S-8 & $\begin{array}{l}\text { A diminuição da audição lhe causa dificuldades para as- } \\
\text { sistir TV ou ouvir rádio? }\end{array}$ & & & \\
\hline E-9 & $\begin{array}{l}\text { Você acha que a dificuldade em ouvir limita, de alguma } \\
\text { forma, sua vida pessoal ou social? }\end{array}$ & & & \\
\hline S-10 & $\begin{array}{l}\text { A diminuição da audição lhe causa dificuldades quando } \\
\text { você está num restaurante com familiares ou amigos? }\end{array}$ & & & \\
\hline
\end{tabular}

Fonte: MATAS, Carla Gentile; IÓRIO, Maria Cecília Martinelli. Verificação e Validação do Processo de Seleção e Adaptação de Próteses Auditivas. In: ALMEIDA, Kátia de; IÓRIO, Maria Cecília Martinelli. Próteses Auditivas: fundamentos teóricos e aplicações clínicas. São Paulo: Lovise, 2003. P. 328.

\section{Recebido em: 09/03/2007}

$1^{a}$ revisão: 02/04/2007

$2^{a}$ revisão: $19 / 07 / 2007$

Aceite final: 11/03/2008 